\title{
Targeting bioelectronically the carotid sinus nerve in Type 2 diabetes: strengths, drawbacks and challenges for the future
}

\author{
Silvia V Conde*,1 \& Maria P Guarino ${ }^{2}$ \\ ${ }^{1}$ CEDOC, NOVA Medical School, Faculdade de Ciências Médicas, Universidade NOVA de Lisboa, Lisboa, Portugal \\ ${ }^{2}$ ciTechCARE, Escola Superior de Saúde de Leiria - Instituto Politécnico de Leiria, Leiria, Portugal \\ *Author for correspondence: Tel.: +351 2188031 00; silvia.conde@nms.unl.pt
}

\begin{abstract}
"We hypothesize that different CSN fibers are coupled with responses to different stimulus and so, the characterization and discrimination of fiber-stimulus associations will allow selective modulation of the fibers at the CSN. If proven right, this hypothesis leads the way to intervene in CSN-mediated metabolic diseases without affecting $\mathrm{O}_{2}$ and $\mathrm{CO}_{2}$ chemosensitivity."
\end{abstract}

First draft submitted: 4 July 2018; Accepted for publication: 20 August 2018; Published online: 22 November 2018

Keywords: bioelectronic modulation • carotid sinus nerve • Type 2 diabetes

\section{Why is the carotid sinus nerve a therapeutic target for bioelectronic modulation in metabolic diseases?}

In recent years, the carotid bodies $(\mathrm{CB})$, classically defined as peripheral sensors for $\mathrm{O}_{2}, \mathrm{CO}_{2}$ and $\mathrm{pH}$, have gained increasing attention due to their function as metabolic sensors implicated in the control of energy homeostasis [1-4]. These tiny organs, located at a privileged anatomic location to signal threats to the central nervous system, are overactivated in insulin resistant, glucose intolerant and hypertensive animal models [5-7] causing an increased adrenergic tone $[5,6]$. Our research group has shown that disturbed CB activity observed in metabolic syndrome and prediabetes $[5,8]$ may be surgically circumvented through resection of the carotid sinus nerve (CSN), which both prevents and restores insulin resistance, glucose intolerance and hypertension in animal models of metabolic syndrome and Type 2 diabetes [5-7]. These positive effects of CSN resection in whole body metabolic homeostasis are mediated by increased glucose uptake and insulin signaling in the liver and in the visceral adipose tissue [6] and by a significant decrease in the heightened sympathetic tone that portrays metabolic diseases [5,6]. Supporting the abovementioned preclinical data, our lab has also shown that intermittent hyperbaric oxygen therapy, frequently used to promote wound healing in diabetic foot ulcers, improves glucose homeostasis in Type 2 diabetes patients [9], an effect that may be attributed to a CB-mediated mechanism, as hyperoxia dramatically reduces peripheral chemoreceptor activity [10]. Therefore, there is currently very strong evidence that CSN neuromodulation may be an appealing alternate approach for patients suffering from metabolic syndrome and early Type 2 diabetes. The attempted therapeutic approaches for CSN neuromodulation, surgery and oxygen therapy, despite being very effective in the disease phenotype, may cause numerous side effects and complications, with varying degrees of seriousness. It has been described that hyperbaric oxygen therapy can frequently produce fatigue and lightheadedness and, more rarely, can cause more severe side effects derived from $\mathrm{O}_{2}$ toxicity as lung damage, rupture of the middle ear, sinus damage and permanent blurred vision [11]. On the other hand, surgical resection of the CSN, apart from being an invasive approach, can lead to side effects related with loss of the peripheral hypoxic response, with decreased sensitivity to $\mathrm{CO}_{2}[12,13]$, impaired response to exercise [14-16] and fluctuations in blood pressure [17]. An alternate approach would be to use a medical device implanted in the CSN, a bioelectronic medicine, with the ability to selectively target disease, by correcting defective action potential patterns without disturbing normal physiological functions. 


\section{Continuous kilohertz frequency alternative current neuromodulation of the carotid sinus nerve}

We recently tested the use of chronic continuous kilohertz frequency alternate current to block CSN transmission and assessed its effects on insulin resistance and glucose intolerance in an early Type 2 diabetes animal model [7]. We demonstrated that bilateral high frequency conduction modulation of the CSN mimics the positive metabolic effects in insulin-resistant, glucose-intolerant animals, previously observed upon CSN bilateral surgical resection, with the advantages of being tunable, nonablative and reversible [7]. The results showed that electrical neuromodulation of the CSN has significant therapeutic potential as a means of controlling CSN activity in metabolic diseases. However, the CSN conveys not only the information that comes from the $\mathrm{CB}$, but also the baroreceptor information that emerges from the carotid sinus and that is involved in the acute adjustment of blood pressure [18]. Despite this, we did not observe significant side effects related to increased blood pressure variability in our experimental setting, the hypothesis that continuous electrical neuromodulation of whole CSN nerve may cause side effects related with blood pressure fine tuning in humans is likely $[17,18]$.

Also, as the $\mathrm{CB}$ is involved in adaptation to hypoxic environments and response to acidosis, adverse effects related with continuous electrical modulation may include exercise intolerance and unsafe hypoxic acclimatization [14-16,19].

\section{Will electrical neuromodulation of carotid sinus nerve be a suitable therapeutic for all Type 2 diabetes phenotypes?}

Although it is widely accepted that metabolic diseases are characterized by increased sympathetic activation, the role of $\mathrm{CB}$ activation as an etiological factor in the different disease phenotypes has yet to be assessed.

Thus, CB functional diagnosis may have predictive value regarding the efficacy of a therapeutic bioelectronic approach directed toward corrective neuromodulation of disturbed CSN discharges. Currently, there is no medical device that synchronizes CB function diagnosis with neuroendocrine homeostasis variables nor clinical guidelines focused on assessing CB function associated with glucose and lipid homeostasis.

The peripheral chemoreceptors, located at the CBs, are intimately involved in cardiorespiratory control, and the assessment of hemodynamic and respiratory variables is the classical way to look at $\mathrm{CB}$ function.

In 1962, Dejours described a way of assessing the 'Respiratory Chemoreflex' measuring the latency of the ventilatory responses after an acute hyperoxic challenge [20]. The Dejours test, as it is still known, was a dynamic respiratory functional test in which baseline respiration was recorded while volunteers breathed $21 \% \mathrm{O}_{2}$ for $45 \mathrm{~s}$, followed by $100 \% \mathrm{O}_{2}$ for $20 \mathrm{~s}$. Respiratory variables were analyzed for $20 \mathrm{~s}$ during $21 \% \mathrm{O}_{2}$ and during the last $15 \mathrm{~s}$ of hyperoxia, to evaluate the sensitivity of the CBs. The amount of the decrement in ventilatory responses, consequent to oxygen breathing, was considered to represent the component of the ventilatory drive attributed to the peripheral chemoreceptors [20]. Although described since 1962, and often used in experimental settings, the Dejours test did not gain clinical relevance due to the scarce knowledge on the pathophysiological involvement of $\mathrm{CB}$ function in clinically relevant diseases. With the new finding that the peripheral chemoreceptors located in the $\mathrm{CBs}$ are involved in the genesis and progression of pandemic nontransmittable diseases, like Type 2 diabetes, the evaluation of the CBs function, as a means to evaluate disease risk, to diagnose subclinical pathologies and to stratify patients for $\mathrm{CB}$ modulatory interventions grows in clinical importance. Additionally, due to the finding that the $\mathrm{CBs}$ also control glucose metabolism and homeostasis, the evaluation of its function must be performed, not only at the cardiorespiratory level, but also in terms of metabolic parameters creating a novel clinical parameter designated as the 'metabolic chemoreflex' that will consist in measuring the latency of the ventilatory responses after an acute mixed meal challenge. The development of a 'CBmeter' that assesses these variables would serve to diagnose patients with altered respiratory and metabolic chemoreflexes, and could be used as a screening tool to detect people with high risk for metabolic diseases and eligible for carotid sinus nerve bioelectronic interventions.

\section{Overcoming the side effects of continuous kilohertz neuromodulation of the carotid sinus nerve}

As the concept of bioelectronic devices targeting the CSN to restore metabolic function is gaining attention and adepts, we have been working in minimizing the off-target effects of neuromodulation of the CSN. Within several specific aims, the one related to identification and characterization of neuronal circuits and signatures that are overactivated within the CSN in metabolic diseases has been the one we have been most dedicated to. We hypothesize that different CSN fibers are coupled with responses to different stimulus and so, the characterization and discrimination of fiber-stimulus associations will allow selective modulation of the fibers at the CSN. If proven 
right, this hypothesis leads the way to intervene in CSN-mediated metabolic diseases without affecting $\mathrm{O}_{2}$ and $\mathrm{CO}_{2}$ chemosensitivity. Additionally, other therapeutic alternatives to continuous high frequency block of the CSN would be to resynchronize action potential firing disease patterns in specific fibers or, in a less targeted approach, to assess the efficacy of intermittent block of the CSN, as an alternative to continuous block, in metabolic homeostasis control.

In conclusion, evidence points toward bioelectronic modulation of the carotid sinus nerve as a robust therapeutic alternative in metabolic syndrome and Type 2 diabetes. The challenges for the future are mainly related to finding an appealing alternative to continuous high frequency alternate current to achieve selective modulation of metabolic responsive CSN fibers with minimal off-target effects.

\section{Financial \& competing interests disclosure}

Silvia Conde and Maria Guarino received funding from GSK Bioelectronics Research Program and are currently funded by Galvani Bioelectronics. The authors have no other relevant affiliations or financial involvement with any organization or entity with a financial interest in or financial conflict with the subject matter or materials discussed in the manuscript apart from those disclosed.

No writing assistance was utilized in the production of this manuscript.

\section{References}

1. Conde SV, Sacramento JF, Guarino MP et al. Carotid body, insulin, and metabolic diseases: unraveling the links. Front. Physiol. 5 , 418 (2014).

2. Conde SV, Ribeiro MJ, Melo BF, Guarino MP, Sacramento JF. Insulin resistance: a new consequence of altered carotid body chemoreflex? J. Physiol. 595, 31-41 (2017).

3. Koyama Y, Coker RH, Stone EE et al. Evidence that carotid bodies play an important role in glucoregulation in vivo. Diabetes 49, 1434-1442 (2000).

4. Wehrwein EA, Basu R, Basu A, Curry TB, Rizza RA, Joyner MJ. Hyperoxia blunts counterregulation during hypoglycaemia in humans: possible role for the role of carotid bodies? J. Physiol. 588, 4593-4601 (2010).

5. Ribeiro MJ, Sacramento JF, Gonzalez C, Guarino MP, Monteiro EC, Conde SV. Carotid body denervation prevents the development of insulin resistance and hypertension induced by hypercaloric diets. Diabetes 62(8), 2905-16 (2013).

6. Sacramento JF, Ribeiro MJ, Rodrigues $\mathrm{T}$ et al. Functional abolition of carotid body activity restores insulin action and glucose homeostasis in rats: key roles for visceral adipose tissue and the liver. Diabetologia 60(1), 158-168 (2017).

7. Sacramento JF, Chew DJ, Melo BF et al. Bioelectronic modulation of carotid sinus nerve activity in the rat: a potential therapeutic approach for Type 2 diabetes. Diabetologia 61(3), 700-710 (2018).

8. Conde SV, Sacramento JF, Guarino MP. Carotid body: a metabolic sensor implicated in insulin resistance. Physiol. Genomics 50(3), 208-214 (2018).

9. Vera-Cruz P, Guerreiro F, Ribeiro MJ, Guarino MP, Conde SV. Hyperbaric oxygen therapy improves glucose homeostasis in Type 2 diabetes patients: a likely involvement of the carotid bodies. Adv. Exp. Med. Biol. 860, 221-225 (2015).

10. Lahiri S, DeLaney RG. Relationship between carotid chemoreceptor activity and ventilation in the cat. Respir. Physiol. 24, 267-286 (1975).

11. Camporesi EM Side effects of hyperbaric oxygen therapy. Undersea Hyperb Med. 41, 253-257 (2014).

12. Dahan A, Nieuwenhuijs D, Teppema L. Plasticity of central chemoreceptors: effect of bilateral carotid body resection on central CO 2 sensitivity. PLoS Med. 4, e239 (2007).

13. Timmers HJ, Wieling W, Karemaker JM, Lenders JW. Denervation of carotid baro- and chemo-receptors in humans. J. Physiol. 553, 3-11 (2003).

14. Forster HV, Pan LG, Bisgard GE, Kaminski RP, Dorsey SM, Busch MA. Hyperpnea of exercise at various PIO2 in normal and carotid body-denervated ponies. J. Appl. Physiol. Respir. Environ. Exerc. Physiol. 54, 1387-1393 (1983).

15. Dempsey JA, Smith CA. Do carotid chemoreceptors inhibit the hyperventilatory response to heavy exercise? Can. J. Appl. Physiol. 19, 350-359 (1994).

16. Forster HV, Pan LG. The role of the carotid chemoreceptors in the control of breathing during exercise. Med. Sci. Sports Exerc. 26, 328-336 (1994).

17. Paton JF, Sobotka PA, Fudim M et al. The carotid body as a therapeutic target for the treatment of sympathetically mediated diseases. Hypertension 61, 5-13 (2013).

18. Marshall JM. Peripheral chemoreceptors and cardiovascular regulation. Physiol. Rev. 74, 543-594 (1994).

19. Gonzalez C, Almaraz L, Obeso A, Rigual R. Carotid body chemoreceptors: from natural stimuli to sensory discharges. Physiol. Rev. 74, 829-898 (1994).

20. Dejours P. Chemoreflexes in breathing. Physiol. Rev. 42, 335-58 (1962). 